\title{
A Storage and Access Architecture for Efficient Query Processing in Spatial Database Systems
}

\author{
Thomas Brinkhoff, Holger Horn, Hans-Peter Kriegel, Ralf Schneider \\ Institute for Computer Science, University of Munich \\ Leopoldstr. 11 B, W-8000 München 40, Germany \\ e-mail: \{brink,holger,kriegel,ralf\}@dbs.informatik.uni-muenchen.de
}

\begin{abstract}
Due to the high complexity of objects and queries and also due to extremely large data volumes, geographic database systems impose stringent requirements on their storage and access architecture with respect to efficient query processing. Performance improving concepts such as spatial storage and access structures, approximations, object decompositions and multi-phase query processing have been suggested and analyzed as single building blocks. In this paper, we describe a storage and access architecture which is composed from the above building blocks in a modular fashion. Additionally, we incorporate into our architecture a new ingredient, the scene organization, for efficiently supporting set-oriented access of large-area region queries. An experimental performance comparison demonstrates that the concept of scene organization leads to considerable performance improvements for large-area region queries by a factor of up to 150 .
\end{abstract}

\section{Introduction}

During the last decade, the management, representation and evaluation of spatial data in information systems gained increasing importance. Geographic information systems (GIS) are increasingly used in public administration, science and business. The nucleus of a GIS is the geographic database system. Contrary to business applications based on standard database systems, such systems are not suitable for geographic applications [Wid 91]. The insufficient expressive power e.g. of relational systems, leads to unnatural data models and to poor efficiency in query processing.

Therefore, various research groups have developed a large number of concepts and techniques for improving single aspects of a geographic database system. Examples are the design of spatial data models or efficient access methods for managing large sets of spatial objects.

In this paper, we will present our geo architecture, a new storage and access architecture for spatial objects integrating several concepts and techniques. It is not our goal to present a new spatial database system or a kernel of a system such as DASDBS [SW 86], EXODUS [CDRS 86], GRAL [Güt 89] and POSTGRES [SR 86]. Instead, we would like to assemble suitable concepts and techniques to a spatial query processing mechanism. One of the most important building blocks of our architecture is the scene organization, a new technique for supporting large range queries. Its performance improvement by up to two orders of magnitude is demonstrated.

The paper is organized as follows. First, we take a closer look at the objects and operations commonly used in geographic information systems. This leads to a set of basic queries which should be efficiently supported by our architecture. A model of spatial query processing using different phases is described in section three. In section four, we present different algorithms and methods for supporting these phases. The new scene organization is described in section 4.4. The integration of the algorithms and methods 
leads to our geo architecture. The rest of the paper contains an investigation of the performance of this architecture. In particular, we present a detailed performance evaluation of our new scene organization for real world data. The paper concludes with a brief statement of our findings and some suggestions for future work.

\section{Objects and operations of a spatial database system}

In this paper, we present a conceptional architecture for storing objects and processing queries in a geographic database system. To develop such an architecture, we first need an exact specification of the objects and queries. This is presented in the following subsections.

\subsection{Objects}

The objects stored in a geographic database are used for modeling specific parts of the surface of the earth with respect to one or several properties. Therefore, the objects are characterized by a spatial and a thematic component. The spatial component describes the spatial locality and the shape of the modeled part of reality whereas the thematic component contains the thematic information.

\section{The spatial component}

The spatial component of an object is represented by one of the basic topological elements of the plane: point, line or area. Points are described by specifying their coordinates with respect to a given coordinate system. For modeling lines, both polylines as well as free-form curves are used. In this paper, we concentrate on representing areas. From the literature two main concepts for representing areas are known: the raster and the vector model. Because of its favorable scaling capabilities, its lower demand of storage and its "object orientation", the vector model has been preferred over the last few years for application in geographic database systems. The type of spatial objects we consider in this paper is the class of simple polygons with holes (SPH for short) (see figure 1). A polygon is called simple if there is no pair of nonconsecutive edges sharing a point. A SPH is a simple polygon where simple polygonal holes may be cut out. The class of SPHs is well suited for geographic applications (see [Bur 86]). It allows representing areas with arbitrary precision and explicitly takes holes into account.

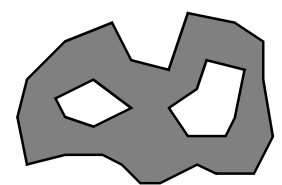

Fig. 1. Simple polygon with holes

The thematic component

The thematic component characterizes an object with respect to one or several thematic properties. We distinguish between qualitative properties such as land use and quantitative properties such as amount of precipitation. For representing thematic values, simple data types such as strings or real numbers are used.

\section{The object model}

The geo architecture to be developed should be able to store sets of objects consisting of a spatial (SPH) and a thematic component (vector of simple data types). Figure 2 gives a typical example of a map which is represented by a set of SPHs. 


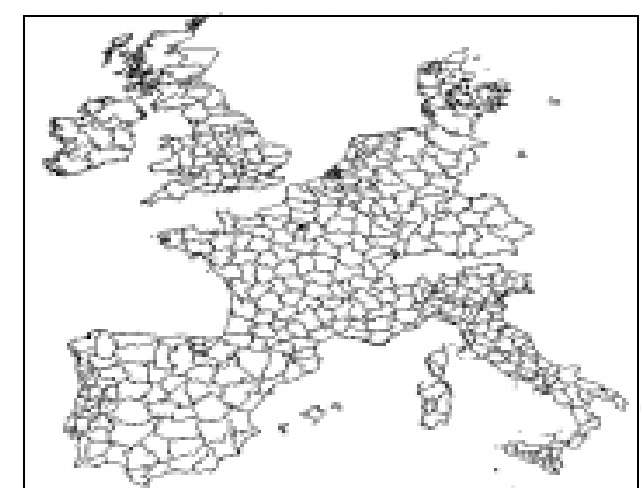

Fig. 2. Map of the European counties modeled by a set of SPH

Both components require a completely different handling by the geo architecture. For managing vectors of simple data types, e.g. in a relational database system, a lot of well known data structures and algorithms are available. However, organizing the spatial component demands for new structures and algorithms. They should organize the objects in such a way that spatial queries referring to location and shape of the objects are processed efficiently.

Additional to these fundamental properties of the spatial objects, two more aspects are important for the design of the geo architecture. First, we need a characterization of the objects from real applications as accurate as possible. Second, we need a specification of the queries and operations to be performed on these objects.

\subsection{Characteristics of the objects}

In this paper, it is not our goal to present a general characterization of the object sets occurring in geographic applications. From our point of view this is impossible because of the very wide application spectrum geographical information systems are used in. Instead, we outline some general properties of the data which influence the design of our geo architecture considerably.

\section{Complexity and variation of the data}

- Number of objects and data volume

In real applications, the number of data objects may be as high as $10^{9}$. The data volume may occupy up to 1 TerraByte (see [Fra 91] and [Cra 90]).

- Variation of objects and sets of objects

Data from real world applications vary extremely with respect to single objects and whole object sets [Fra 91]. This particularly refers to the following aspects:

- Object extensions

It varies in a range of $1: 10^{6}$ [Fra 91], where the largest objects may occupy the whole data space.

- Object shape

- Amount of storage

As an example, in the World Data Bank II [GC 87] the amount of storage for one polygonal object varies between $0.5 \mathrm{~KB}$ and more than $1.1 \mathrm{~GB}$. 
- Distribution of the objects in the data space

The number of objects per unit (density) varies in a range of $1: 10^{4}$ in real world applications [Fra 91].

In particular, we have to consider that there are no upper bounds neither for the extension of objects, the complexity of object structure, the amount of storage, nor for the density of the objects.

\section{Persistent storage of the objects in a weak dynamic environment}

Recording the data of a geographic information system is an expensive task. Very often, data from paper maps as well as satellite pictures have to be integrated into a seamless database. This work is often a source of inaccuracy and inconsistency, which has to be revealed and removed by using time consuming consistency check mechanisms. Altogether recording the data and preserving consistency of the data account for approximately $80 \%$ of the operating costs of a geographic database system [Aro 91].

After recording the database, it is persistently stored and used on a long term basis. However, the database is not static because correcting mistakes, removing inconsistencies and adapting to changes in the real world leads to updates of the data. All in all, the database is weakly dynamic.

The properties of spatial objects mentioned above and the queries and operations described in the following section form a requirement definition for the geo architecture which is described in detail in section 4

\subsection{Queries and operations}

Geographic database systems are used in very different application environments. Therefore, it is not possible to find a compact set of spatial queries and operations fulfilling all requirements of geographic applications [SV 89]. Instead, we present four basic classes of operations each with a number of typical representatives which should be supported by our architecture.

\section{1) Modifications}

Analogously to standard database systems, there are operations for insertion, deletion and update of records in a geographic database system.

\section{2) Selections}

We can distinguish between two types of selections: those referring to the spatial and those referring to the thematic component of an object.

\section{a) Spatial selections:}

- Point Query

Given a query point $P$ and a set of objects $M$. The point query yields all the objects of $M$ geometrically containing P (see figure 3(a)).

- Region Query

Given a polygonal query region $R$ (of type SPH) and a set of objects $M$, the region query yields all the objects of $M$ sharing points with $R$. A special case of the region query is the window query. The query region of a window query is given by a rectilinear rectangle (see figure 3(b)). Both, the window query and the region query are often called range queries. 

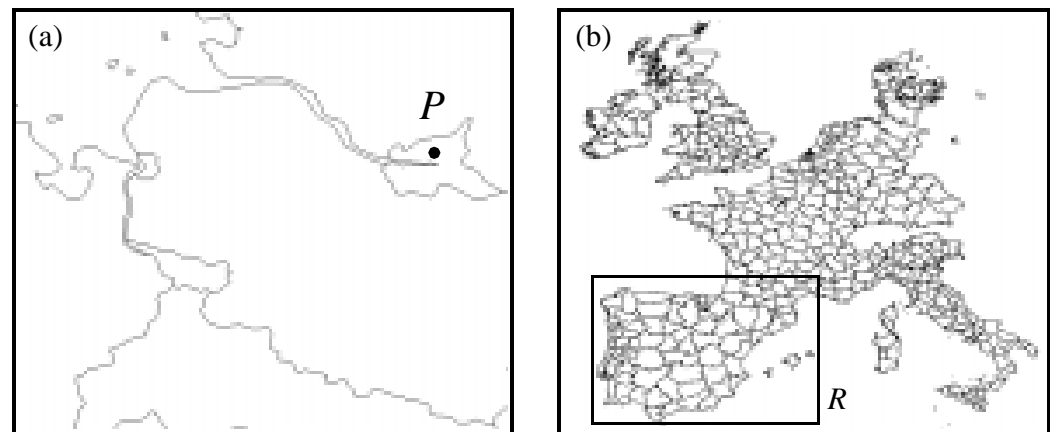

Fig. 3. Examples for a point and a window query

\section{b) Thematic Selections:}

When performing a thematic (relational) selection the objects are selected with respect to properties of their thematic component. Within this section, we pay attention only to the spatial component of the objects. In section 4.5 we will describe how to support thematic selections.

\section{3) Combinations}

- Spatial Join

For two given object sets A and B the spatial join operation yields all pairs of objects ( $a, b), a \in A, b \in B$ whose spatial components intersect. More precisely, for each object $a \in A$ we have to look for all objects in B intersecting with $a$. Note, that for efficient processing of the spatial join a selective spatial access to the objects is necessary.

\section{- Map Overlay}

The map overlay is one of the most important operations in a geographic information system [Bur 86]. It combines two or more sets of spatial objects. This combination is controlled by the overlay function determining in which way intersecting objects have to be handled. The map overlay is completely based on variants of the spatial join operation. In addition to the spatial join, the intersection of a pair of overlapping objects has to be computed. Neighboring objects with identical values of their thematic component should be merged [KBS 91].

\section{4) Analyzing sets of objects}

Selections or combinations of existing sets of objects are often followed by further processing steps in practical applications. The operations and algorithms used for these steps are very specific for a particular application and, therefore, are not supported by a general storage and access architecture. Without considering the details, we can distinguish two classes of these operations and algorithms.

\section{- Automatic analysis}

Analyzing functions applied to the spatial and/or the thematic component of the objects are part of this class. Typical representatives are: calculating the average of the area or perimeter of a set of objects, calculating the minimum and maximum of thematic attributes etc.

\section{- Visualization}

In many cases the automatic analysis of a database is not possible and manual inter- 
mediate steps performed by a user are necessary to complete the analysis. For this purpose, a visualization of the data on a graphic device is necessary.

The above mentioned facts clearly demonstrate that spatial selections are of great importance within the set of spatial queries and operations. They do not only represent an own query class, but also serve as a very important basis for the operations of the classes 2 - 4. Therefore, an efficient implementation of spatial selections is an important requirement for good performance of the complete geographic information system.

\section{A phase model for geometric query processing}

After the description and specification of objects and queries, we will design an architecture for storing spatial objects and efficiently processing queries. The main task of the architecture is the efficient processing of spatial queries and operations. Therefore, in this section, we take a closer look at this type of queries, distinguish different phases in their processing and specify algorithms and data structures for their processing.

As mentioned in the last section, spatial selections are the most important basic operation in spatial query processing. Their execution can be described abstractly as a sequence of steps:

\section{Step 1: Scaling down the data space}

Considering spatial selections in more detail, it turns out that only a local part of the complete data space has to be investigated. Only this area contains candidate objects that may fulfill a selective query.

For an efficient scaling down of the data space, it is essential to use data structures organizing the objects with respect to their spatial locality and shape. Obviously, objects jointly fulfilling a query condition lie close together in the data space. Therefore, a physical clustering of the objects with respect to their spatial locality and shape is essential for providing efficient spatial query processing.

Due to the arbitrary complexity of real geographic objects, it is not possible to build up an index considering the complete information on the extension of the objects. Thus, the access method is not able to yield the exact result of a query. Instead, it excludes a large subset of objects from the result. A set of candidate objects that may fulfill the query condition remains and has to be passed on to step 2 of the query processing mechanism. Orenstein established in [Ore 89] the terms filtering and refinement for this type of query processing.

\section{Step 2: Exact investigation of the objects}

Step 2 of the query processing tests whether a candidate object actually fulfills the query condition or not. For that purpose, a spatial predicate, e.g. "polygon contains point" or "rectangle intersects polygon", has to be checked. Similar to step 1, this test consists of different phases. First, the test has to be restricted to only that part of the object that is really relevant for the test. Figure 4 gives an example: To evaluate whether the query window $R$ overlaps Lake Volta, only its northern west peak has to be examined.

Due to the complexity of the objects on the one hand and the selectivity of spatial queries on the other hand, it is useful to structure the objects locally. The resulting structure elements have to be organized in a data structure referring to their spatial locality and extension. Using this data structure, we can efficiently decide which parts of the object are actually relevant to the query. Only this small number of local parts is further 
examined using computational geometry algorithms, which finally decide whether an object fulfills the query or not.

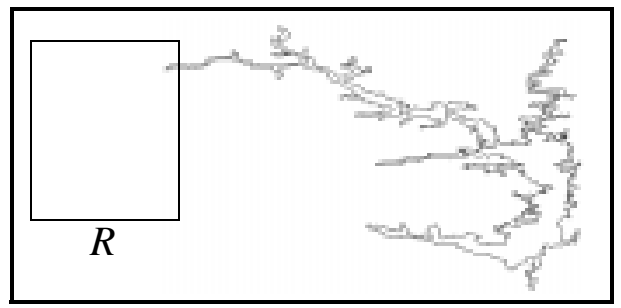

Fig. 4. Test of a query window against Lake Volta

\section{Step 3: Output of objects for further processing}

After identifying an object as part of the result, it is usually passed on to further processing e.g. analyzing steps, output operations etc. Therefore, a physically connected storage of all parts of the objects is necessary to support a fast access to the complete object.

\section{An architecture for query processing in spatial database systems}

After the abstract description of the phase model for spatial query processing, we present algorithmic techniques for supporting the individual phases. Later on in this section, these techniques are used as building blocks within our geo architecture.

\subsection{Spatial access methods}

Access methods as an essential part of the internal level of a database system are used to organize a dynamic set of objects on secondary storage. One-dimensional access methods like $B$-trees or linear hashing are not suitable for spatial database systems. For these systems, we have to look for data structures which organize the polygonal objects with respect to their location and extension in the data space. The arbitrary complexity of the spatial objects (simple polygon with holes) makes it very difficult to develop a structure considering the whole object description. Instead, we consider access methods for simpler two-dimensional objects. Surveys of spatial access methods can be found e.g. in [Sam 90] and [Wid 91].

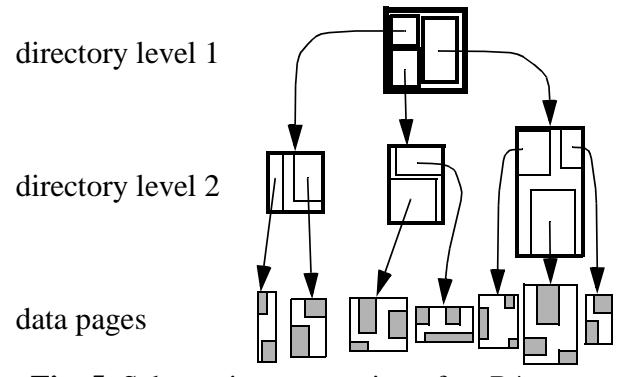

Fig. 5. Schematic presentation of an $\mathrm{R}^{*}$-tree

The simplest class of two-dimensional objects are rectilinear rectangles. For this class of objects, a number of index structures already exists. A popular representative is the $R$-tree [Gut 84]. The R-tree stores as many spatially close objects (rectangles) on one data page as it accommodates and surrounds them by their minimum bounding box. A 
set of such bounding boxes is stored on a (directory) page. Again, their minimum bounding box is computed and stored in a directory page one level above and so on. In this way, the whole object set is stepwise spatially clustered and a tree-like directory is created (see figure 5).

A very efficient version of the R-tree is the $R^{*}$-tree [BKSS 90]. Within this data structure sophisticated algorithms for page splitting and local reorganizations are used. The overlap of page regions and the length of their margin are minimized as well as the dead space, i.e. the space occupied unnecessarily by page regions.

This idea of organizing rectangles leads to an efficient processing of point queries and small window queries [BKSS 90]. Unfortunately, this is restricted to rectangles or other simple spatial objects, not larger than a data page. In real applications, it is absolutely necessary to store more complex objects and to process large window queries efficiently. Later on in this section, we will present an access architecture for managing arbitrary simple polygons with holes and processing large window queries efficiently.

\subsection{Approximations}

The set of results to a spatial query consists of all the objects fulfilling a geometric predicate e.g. containing a query point. As mentioned in the last section, spatial access methods are used for excluding a large subset of the objects from the result as early as possible. The remaining candidate objects have to be investigated by computational geometry algorithms. Considering complex objects (polygons with large numbers of vertices), this is a time consuming task. This leads to the idea of a geometric pretest. Such a test should be easy to process and should decide for a large number of objects whether they fulfill the query condition or not.

For implementing the idea of a geometric pretest, the concept of object approximations is an adequate approach. In [Kri 91a] a detailed classification of different approximation techniques is given. The description of an approximation should be simple and its quality should be high, two obviously competing criteria. To make object approximations useful for a geometric pretest, the object has to be contained completely in its approximation (conservative approximation) [Sch 92]. Examples for conservative approximations are minimal bounding boxes, convex polygons, ellipses etc. (figure 6).

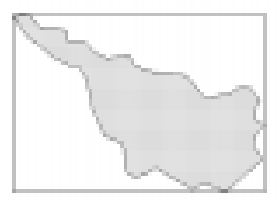

minimal bounding box

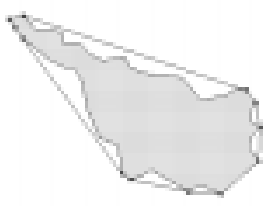

convex hull

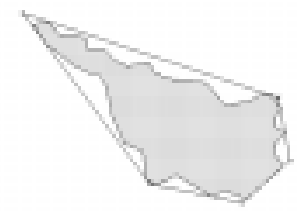

5-corner

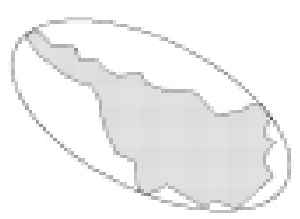

ellipse

Fig. 6. Various conservative approximations

Let us have a closer look at the processing of a point query using object approximations. First, for all candidate objects it is tested, whether their approximation contains the query point or not. In case of a negative result, the object does not contain the query point either. The object is discarded and a time consuming point-in-polygon test could be saved. Only in case of a positive pretest, the object itself has to be tested.

[BKS 93a] contains a detailed examination of object approximations used for spatial query processing in a real data environment. It turned out that the convex 5-corner is the best compromise between the approximation quality and storage amount. Using the 
$\mathrm{R}^{*}$-tree, it is shown that other approximations than the minimal bounding box can efficiently be organized in a spatial access method originally designed for bounding boxes.

\subsection{Object decompositions}

Object approximations are applied to avoid complex geometric tests. Object decomposition techniques, however, are used to simplify and speed up their processing.

Consider again a point-in-polygon test. For processing this test an algorithm with linear runtime complexity is necessary [PS 88]. This examination of complex polygons i.e. polygons with thousands of vertices consumes a considerable amount of CPU time. On the other hand, only a small local part of the object is actually relevant for the decision whether an object contains a point or not. This leads to the idea of object decomposition. Applying this idea, the objects are divided into a number of simple and local components, e.g. triangles, convex polygons etc.. During spatial query processing, only one or a small number of these components has to be checked. In [KHS 91] and [Kri 91a] the decomposition approach for simple polygons with holes is presented and discussed in detail.

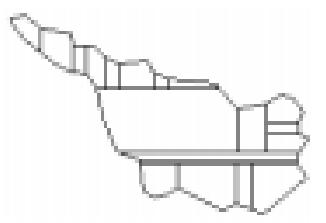

convex polygons

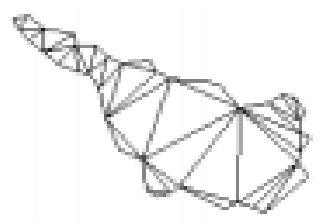

triangles

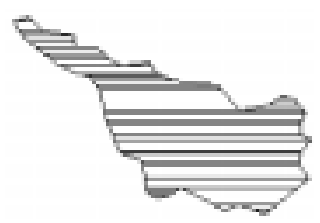

trapezoids

Fig. 7. Three decomposition techniques for simple polygons

Using object decompositions geometric tests are applied only to components, e.g. trapezoids, which is much more efficient than testing the whole polygon. To decide which components are relevant for a particular test, we use again an $\mathrm{R}^{*}$-tree to organize the components of one object with respect to their location and shape. The resulting tree is called a $T R^{*}$-tree. In [SK 91] we demonstrated that the TR*-tree efficiently supports various types of spatial queries and operations.

\subsection{Scene organization}

One important requirement for geographic database systems is the set orientation [Wid 91]. A spatial query processor has to perform small queries as well as large queries efficiently. When processing a large query, a large amount of data is transferred from secondary storage into main memory. The concepts presented up to now in this paper, merely support an efficient processing of small queries but do not speed up large queries considerably. Therefore, there is an obvious demand for a concept supporting set orientation.

Considering the existing storage organization and the type of objects to be stored, we can observe the following points:

- The objects are very large in comparison to the size of the pages they are stored in. Even in the case of large pages (e.g. 4 KByte), the number of objects per page is usually small and often we need several pages for storing just one single object.

- The pages used for storing objects are distributed on the secondary storage device independently from spatial aspects, i.e. pages lying adjacent in space lose their neighborhood on the storage device. Large region queries transfer a large amount of 
spatially adjacent pages into main memory. Therefore, an arbitrary distribution of these pages on the disk leads to very high access costs during query processing.

The concepts presented in the sections before preserve only a local ordering within the pages [Wid 91]. To support the set orientation in an appropriate way, a global order preservation i.e. a physical clustering of larger storage units, is required.

Different approaches are conceivable to handle larger storage units. In [Wei 89] using larger pages, pages of variable length, various buffering strategies and physical clustering of pages combined with a set-oriented interface are discussed in detail to handle large complex objects. In this paper, physical clustering of pages is favored and naturally offers itself as an adequate approach to store scenes within our geo architecture. To translate this approach into action, we need a set-oriented interface between the database system and the secondary storage device [Wei 89]. Such an interface allows an efficient transfer of physically adjacent pages from secondary storage to main memory. The implementation of such an interface is not the subject of this paper.

In [HSW 88] an idea based on dynamic z-hashing for implementing physical clustering of pages is presented. This idea is applied to rectangles in [HWZ 91]. However, the global order is preserved only for approximations of objects. Furthermore, this hash approach is not applicable to access methods with an arbitrary space partitioning scheme. Therefore, we have developed a concept based on the partitioning scheme of the $\mathrm{R}^{*}$-tree.

\section{Building up the scene organization}

As mentioned before, we use the $\mathrm{R}^{*}$-tree as a major component of our geo architecture, due to its good performance and its robustness. The $\mathrm{R}^{*}$-tree uses a very efficient scheme for space partitioning neither clipping nor transforming the spatial objects. These facts lead to the idea of using the partitions i.e. subtrees of the $\mathrm{R}^{*}$-tree as basic units for physical clustering. In the following, a scene is defined as a subtree of the $\mathrm{R}^{*}$-tree physically clustered on secondary storage. One scene consists of a large set of physically adjacent pages containing all corresponding objects. Using this approach, no additional data structure for handling scenes is necessary.

An object larger than one page is stored on several pages such that all of them are physically clustered within one scene. Thus, also the transfer of such a large object into main memory is supported by the scene organization (see step 3 of the phase model). Note that no order has to be preserved within each scene.

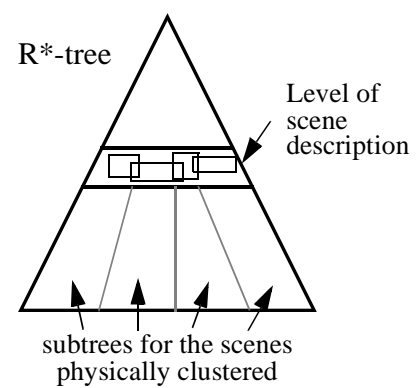

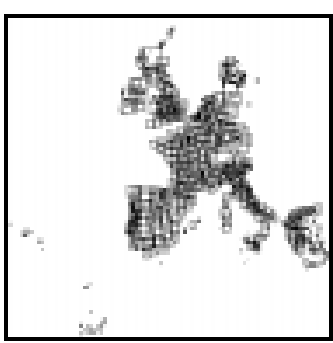

(a) approximations

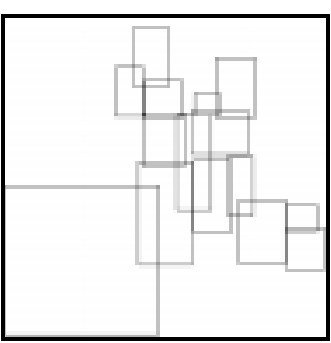

(b) scenes

Fig. 8. Scene organization

In addition to a schematic structure of the scene organization, figure 8 presents the particular scene organization for the counties of the European Community (see figure 1 
also). The $\mathrm{R}^{*}$-tree contains the polygons representing the counties, their decomposition components and their approximations (figure 8 (a)). Figure 8 (b) depicts the partitioning of the $\mathrm{R} *$-tree on a higher directory level. The rectangles describe the scenes, the corresponding subtrees are physically clustered.

\section{Query processing}

Using our scene architecture, small queries as well as large queries are processed efficiently. Small queries are processed by single page accesses as described before. If a range query specifies a larger query region, all scenes intersecting the query region, i.e. subtrees of the $\mathrm{R}^{*}$-tree, are transferred into the main memory. For each scene just one search operation on secondary storage is necessary. Without a scene organization, we need one search operation for each page which is much more expensive. Unfortunately, a scene may contain a number of objects not fulfilling the query condition (false hits). Nevertheless, the false hits are also transferred into main memory. A relatively small number of false hits does not affect performance considerably, since the time needed for searching a page drastically exceeds the time for transferring a page [PH 90]. In addition, the degree of intersection between the scene and the query region may be used as a measure to decide whether the scene is transferred completely or whether the query is answered without using the scene organization. A detailed performance evaluation of the scene organization is presented in section 5.1.

After transferring the scene into main memory, a query is processed as usual, i.e. using approximations and decomposition techniques (see section 4.2 and section 4.3). A detailed algorithmic description of the dynamic organization of the scene architecture is presented in [Sch 92] and [BKS 93b]. Supplementing the presented query processing techniques by a scene organization allows an efficient query processing for queries of arbitrary size.

\subsection{Integration of thematic attributes}

The techniques presented up to now are completely dedicated to spatial queries. Queries referring to thematic attributes of the stored objects are also important in geographic information systems (see section 2.1).

For an efficient support of thematic queries, an additional index, i.e. a secondary index (e.g. a B-tree) is necessary for the relevant thematic attributes. The $\mathrm{R} *$-tree in cooperation with the scene organization determines the location of physical storage of the objects. For connecting both, we need a link table. This table assigns to each spatial object, which is represented by a unique surrogate one data page of the $\mathrm{R}^{*}$-tree. If the data page of the spatial object changes, only the entry of the link table has to be updated. An update of the secondary index is not necessary. To allow an access from the spatial index to the link table, all entries of the spatial objects in the data pages have to be extended by a surrogate.

In figure 9, the integration of a secondary index and a link table into our complete geo architecture is presented (for more details see also [Kri 91b] and [Sch 92]).

\subsection{The geo architecture}

Up to now, we have presented basic concepts and techniques for an efficient query processing in geographic databases. The goal of this section is the integration of these concepts into our geo architecture. This architecture is presented in figure 9.

The basic building block of our architecture is the $\mathrm{R}^{*}$-tree. It organizes the objects on secondary storage pagewise and allows an efficient spatial indexing. Starting with 
the root, a spatial query passes through the $\mathrm{R}^{*}$-tree, thereby locating one or several scene descriptions. If the intersection of the query region and the scene exceeds a given threshold, the scene is completely transferred into main memory where query processing proceeds. Otherwise, the required data regions are transferred page by page.

The next ingredient of the architecture are approximations. They support a first preselection to determine whether an object fulfills the query or not. For that purpose, the approximations, e.g. minimal bounding 5-corners, of the objects are stored in the entries of the data pages. If the approximation of a spatial object fulfills the query, the object itself has to be further investigated. Therefore, each object entry contains a pointer to its exact geometric representation managed by a TR*-tree. The TR*-tree organizes all decomposition components and helps exploiting spatial selectivity in query processing. Instead of applying time consuming computational geometry algorithms to complete spatial objects, the query condition is evaluated just considering simple components.

The architecture is completed by secondary indices for thematic attributes. A thematic query traverses the B-tree yielding one or more surrogates. These surrogates are used for accessing to the link table providing the number of the data page storing the object entry.

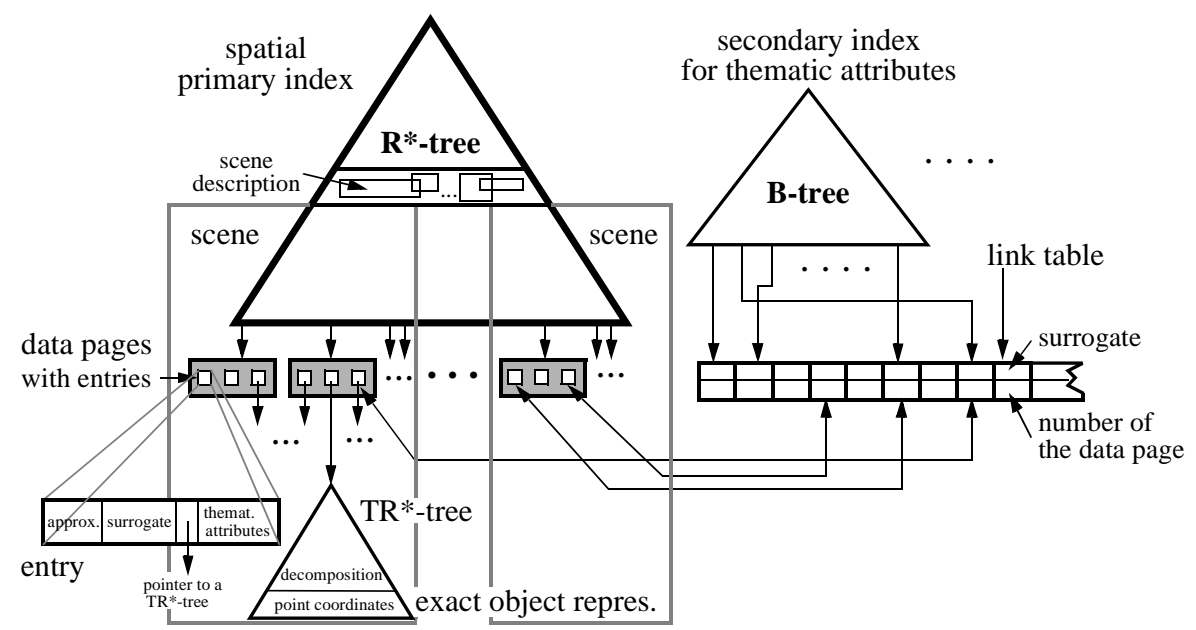

Fig. 9. Integration of efficient building blocks into our geo architecture

\section{Evaluation}

The techniques integrated in our architecture for spatial databases have been investigated and tested extensively. The basic component of the architecture is the $\mathrm{R}^{*}$-tree. In [BKSS 90] a detailed performance evaluation is presented and it turns out that the $\mathrm{R} *$-tree outperforms the other R-tree variants. A performance comparison of the $\mathrm{R}^{*}$-tree, the $\mathrm{R}^{+}$-tree and the PMR-Quadtree is presented in [HS 92]. Various approximations are compared in [BKS 93a]. The minimum bounding 5-corner turns out to be best suited for spatial query processing.

The decomposition approach is examined in [Kri 91a] and [KHS 91]. Especially small queries are processed much faster using the convex and the trapezoid decompo- 
sition instead of the undecomposed representation. The integrated representation of a polygon decomposed into trapezoids using a TR*-tree is considered in [SK 91].

The combination of spatial objects to scenes is introduced in this paper for the first time. As mentioned before, we expect a considerable performance improvement by using this approach. This expectation is confirmed by a detailed performance evaluation of the scene architecture presented in the following subsection.

\subsection{Evaluation of the scene organization}

Basically, there are three different models for storing spatial objects:

1.) Storing the exact object representations outside of the data pages (model 1) In the data pages of the index structure, we store the approximations and the pointers to the exact representations of the objects. The exact representation is stored outside the index structure, e.g. in a sequential file. This approach is used in quadtrees for instance [HS 92]. In other words, the spatial index structure is a primary index for the approximations and a secondary index for the spatial objects. This model is shown schematically in figure 10. The main advantage of this scheme is the large number of approximations stored together in one data page, i.e. a maximum degree of local ordering of the approximations is preserved. Furthermore, there is no limit to the size of the exact object representation. A fundamental drawback is the fact that the order preservation just refers to the object approximations and not to the objects themselves. Consequently, when processing range queries for each access to an exact object representation an additional page access is necessary.

2.) Storing the exact object representation inside the data pages (model 2)

The exact representation of the objects is stored, in addition to the approximations, inside the data pages. Therefore, spatial neighborhood is physically preserved and objects are transferred into main memory just using one disk access [Wid 91]. In contrast to the first model, the index structure is a primary index for the spatial objects and determines their storage location. An essential drawback of this approach is the low number of objects fitting into one page. As a consequence, neighboring objects are often stored in different pages. In section 2.2 we have emphasized that objects larger than one data page often occur in geographic databases. Handling these objects with the second model is a difficult task because a special page overflow mechanism has to be implemented.

3.) Storing objects in a scene organization (model 3)

This model has already been presented in section 4.4. Larger parts of the data are physically clustered within so called scenes and organized in an $\mathrm{R} *$-tree.

In figure 10 the three models are depicted.

The scene organization has been designed for supporting large region queries. Considering such set-oriented queries, we have to take a closer look to two important problems:

- Which performance is gained by the three models ? Is the performance of the scene organization superior to the other two models ?

- Which size of the scenes leads to the best query performance ? Does this size significantly depend on the size of the range queries? 


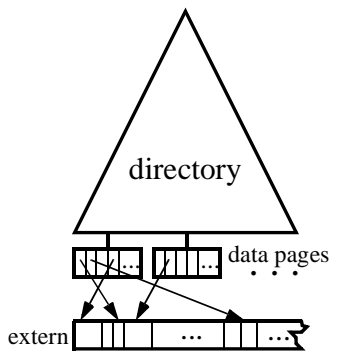

model 1

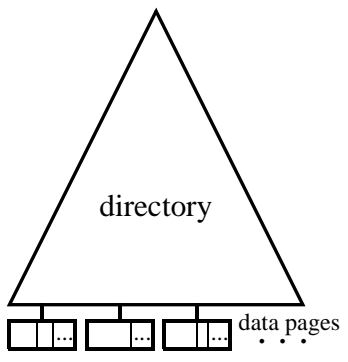

model 2

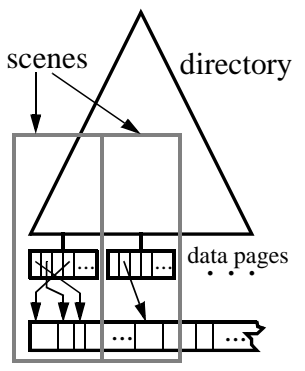

model 3

Fig. 10. Models for storing spatial objects

\section{Test environment}

To find an answer to these questions, we have carried out a detailed empirical performance comparison of the three models. We used real test data from the US Bureau of the Census [Bur 89] containing county borders, highways, railway connections and rivers of four Californian counties. This database consists of 119.151 lines, each consisting of 2 to 349 points. Each co-ordinate is represented by a real number of 8 Bytes. Altogether the database has a size of 15.9 MByte. The lines were approximated by using minimal bounding boxes. For the representation of these boxes 16 Bytes are available. These boxes are depicted in figure 11 (a).

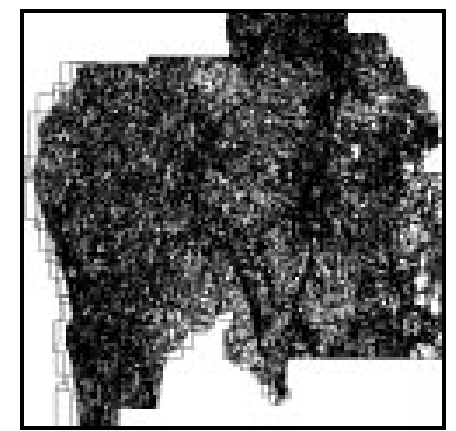

(a) Test data

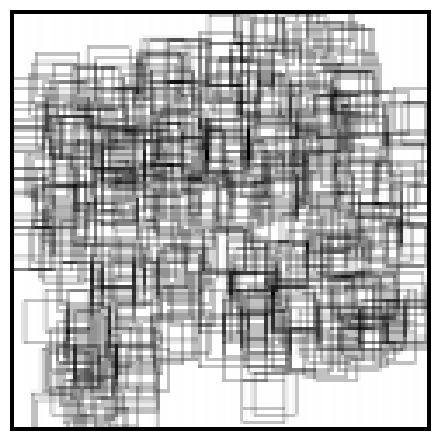

(b) Query regions (1\% area)

Fig. 11. Data and queries used for the tests

Using this data set we built up three $\mathrm{R}^{*}$-trees referring to the three different models. The page capacity was 4 KByte.

To investigate the performance of the models for large query regions, we carried out four test series with different sizes of the query regions. Each series consists of 464 quadratic window queries uniformly distributed over the data space covered by the objects. The area of the query regions varies between $0.25 \%$ and $16 \%$ of the data space. In figure 11 (b) the $1 \%$ queries are shown. Table 1 presents the query specification of the four test series. 


\begin{tabular}{|c||c||c|c||c|c|}
\hline \multicolumn{1}{|c||}{} & \multicolumn{2}{|c||}{$\begin{array}{c}\text { size of } \\
\text { the queries } \\
\text { test series }\end{array}$} & \multicolumn{2}{c||}{ per test series } & \multicolumn{2}{c|}{ average per query } \\
\cline { 3 - 6 } & $\begin{array}{c}\text { (pe data space) } \\
\text { the }\end{array}$ & $\begin{array}{c}\text { number } \\
\text { of records }\end{array}$ & $\begin{array}{c}\text { data volume } \\
\text { (KByte) }\end{array}$ & $\begin{array}{c}\text { number } \\
\text { of records }\end{array}$ & $\begin{array}{c}\text { data volume } \\
\text { (KByte) }\end{array}$ \\
\hline \hline I & $0.25 \%$ & 189,229 & 29,392 & 408 & 63 \\
\hline II & $1 \%$ & 714,937 & 105,521 & 1,541 & 227 \\
\hline III & $4 \%$ & $2,687,648$ & 382,483 & 5,792 & 824 \\
\hline IV & $16 \%$ & $9,462,455$ & $1,315,236$ & 20,393 & 2,835 \\
\hline
\end{tabular}

Tab. 1. Characteristics of the test series

To evaluate the performance of the three models, we need a measure for the access cost. The time necessary for reading one page into main memory consists of the search time, i.e. the time needed for locating the page on secondary storage, and the transfer time, i.e. the time needed to transfer the data from secondary into main memory. Normalize the cost for a transfer operation to 1 . Then in real magnetic disk drives the cost for a search operation is approximately 10 [PH 90]. If $\mathrm{N}_{\mathrm{S}}$ denotes the number of search operations and $\mathrm{N}_{\mathrm{T}}$ denotes the number of transfer operations then the complete access cost $\mathrm{A}$ is given by:

$$
A=10 N_{S}+N_{T}
$$

Considering range queries, the access cost within the $\mathrm{R}^{*}$-tree is negligible in comparison to the access cost of the exact object representation. Thus, in the following, we take into account only the access cost for reading the exact object representation.

\section{Test results:}

In table 2, we present the access cost when storing the lines outside the data pages (model 1). The number of search operations $\left(\mathrm{N}_{\mathrm{S}}\right)$, the number of transfers $\left(\mathrm{N}_{\mathrm{T}}\right)$ and the access cost $A$ are presented (in the following table, $A$ is not directly calculated from $N_{S}$ and $\mathrm{N}_{\mathrm{T}}$ due to rounding).

\begin{tabular}{|c|c|c||c|c|c||c|c|c||c|c|c|}
\hline \multicolumn{3}{|c||}{ test series I $(0,25 \%)$} & \multicolumn{3}{|c||}{ test series II (1\%) } & \multicolumn{3}{|c|}{ test series III (4\%) } & \multicolumn{3}{|c|}{ test series IV (16\%) } \\
\hline $\mathrm{N}_{\mathrm{S}}$ & $\mathrm{N}_{\mathrm{T}}$ & $\mathrm{A}$ & $\mathrm{N}_{\mathrm{S}}$ & $\mathrm{N}_{\mathrm{T}}$ & $\mathrm{A}$ & $\mathrm{N}_{\mathrm{S}}$ & $\mathrm{N}_{\mathrm{T}}$ & $\mathrm{A}$ & $\mathrm{N}_{\mathrm{S}}$ & $\mathrm{N}_{\mathrm{T}}$ & $\mathrm{A}$ \\
\hline \hline 189 & 189 & 2,082 & 715 & 715 & 7,864 & 2,688 & 2,689 & 29,585 & 9,462 & 9,463 & 104,088 \\
\hline
\end{tabular}

Tab. 2. Access cost for model 1 (in thousand, rounded)

Storing the exact object representation outside the data page, requires at least one (expensive) search operation for each answer, because of the missing spatial organization of the exact object representations.

Table 3 contains the results for model 2, i.e. for storing the lines inside the data pages.

\begin{tabular}{|c|c|c||c|c|c||c|c|c||c|c|c|}
\hline \multicolumn{3}{|c||}{ test series I $(0,25 \%)$} & \multicolumn{3}{|||}{ test series II $(1 \%)$} & \multicolumn{3}{|c|}{ test series III (4\%) } & \multicolumn{3}{|c|}{ test series IV $(16 \%)$} \\
\hline $\mathrm{N}_{\mathrm{S}}$ & $\mathrm{N}_{\mathrm{T}}$ & $\mathrm{A}$ & $\mathrm{N}_{\mathrm{S}}$ & $\mathrm{N}_{\mathrm{T}}$ & $\mathrm{A}$ & $\mathrm{N}_{\mathrm{S}}$ & $\mathrm{N}_{\mathrm{T}}$ & $\mathrm{A}$ & $\mathrm{N}_{\mathrm{S}}$ & $\mathrm{N}_{\mathrm{T}}$ & $\mathrm{A}$ \\
\hline \hline 16 & 16 & 175 & 52 & 52 & 573 & 180 & 180 & 1,985 & 610 & 610 & 6,710 \\
\hline
\end{tabular}

Tab. 3. Access cost for model 2 (in thousand, rounded) 
Compared to model 1, model 2 needs considerably less search operations. The reason for this behavior is the fact that many neighboring objects are stored in just one data page and read into main memory by one access. The improvement only marginally depends on the size of the query ranges. $\mathrm{N}_{\mathrm{T}}$ has basically the same size as $\mathrm{N}_{\mathrm{S}}$, because in the test data only a few records are larger than one page.

In the scene organization (model 3), the results considerably depend on the average size of the scenes. Using model 1, the exact object representation is only accessed if it is necessary for query processing. Contrarily, in the second model exact object representations are read into the main memory if they are close to the margin of the query region, but do not intersect the query region (false hits). Large scenes need only a small number of search operations but a high number of transfers from the secondary to the main memory due to the large number of false hits. On the other hand, the smaller the scenes, the higher the effort for searching and the lower the number of transfers. To examine this effect in more detail and to determine the optimal scene size, we varied the scene size in our comparisons. The results are presented in table 4 where the best results are shaded.

\begin{tabular}{|c|c|c|c|c|c|c|c|c|c|c|c|c|}
\hline \multirow{2}{*}{$\begin{array}{c}\text { average } \\
\text { scene size } \\
\text { (Byte) }\end{array}$} & \multicolumn{3}{|c|}{ test series I $(0.25 \%)$} & \multicolumn{3}{|c|}{ test series II $(1 \%)$} & \multicolumn{3}{|c|}{ test series III (4\%) } & \multicolumn{3}{|c|}{ test series IV (16\%) } \\
\hline & $\mathrm{N}_{\mathrm{S}}$ & $\mathrm{N}_{\mathrm{T}}$ & A & $\mathrm{N}_{\mathrm{S}}$ & $\mathrm{N}_{\mathrm{T}}$ & A & $\mathrm{N}_{\mathrm{S}}$ & $\mathrm{N}_{\mathrm{T}}$ & A & $\mathrm{N}_{\mathrm{S}}$ & $\mathrm{N}_{\mathrm{T}}$ & A \\
\hline $1,852,750$ & 1.1 & 698 & 709 & 1.2 & 781 & 794 & 1.6 & 943 & 959 & 2.1 & 1,666 & 1,188 \\
\hline 757,943 & 1.2 & 275 & 287 & 1.6 & 343 & 345 & 2.4 & 505 & 529 & 4.1 & 837 & 877 \\
\hline 273,357 & 1.5 & 128 & 144 & 2.3 & 185 & 208 & 4.2 & 324 & 365 & 8.6 & 638 & 725 \\
\hline 140,124 & 1.8 & 78 & 96 & 3.0 & 123 & 153 & 6.0 & 238 & 298 & 14.1 & 528 & 669 \\
\hline 91,619 & 2.2 & 62 & 84 & 3.9 & 103 & 142 & 8.5 & 214 & 299 & 20.8 & 503 & 711 \\
\hline 79,027 & 2.1 & 51 & 72 & 3.9 & 90 & 130 & 8.8 & 191 & 280 & 22.7 & 474 & 701 \\
\hline 63,402 & 2.3 & 46 & 70 & 4.5 & 85 & 130 & 10.4 & 187 & 291 & 27.5 & 467 & 742 \\
\hline 33,283 & 3.2 & 36 & 68 & 6.7 & 70 & 137 & 17.3 & 167 & 340 & 48.8 & 447 & 936 \\
\hline 18,610 & 4.2 & 26 & 68 & 10.0 & 57 & 158 & 27.8 & 151 & 429 & 82.8 & 432 & 1,260 \\
\hline 10,716 & 6.1 & 23 & 84 & 15.4 & 54 & 209 & 45.6 & 153 & 609 & 140 & 452 & 1,853 \\
\hline 8,367 & 6.8 & 21 & 87 & 18.2 & 52 & 235 & 55.6 & 152 & 708 & 175 & 460 & 2,210 \\
\hline
\end{tabular}

Tab. 4. Access cost for the scene organization (model 3) (in thousand, rounded)

As expected, with increasing scene size $\mathrm{N}_{\mathrm{S}}$ decreases and $\mathrm{N}_{\mathrm{T}}$ increases. Scene sizes between 25 and $100 \mathrm{KByte}$ lead to minimum access cost, depending on the size of the queries. The larger the queries, the larger the optimal scene size. However, this dependency is not as strong as expected. There is a factor of 64 in the size of the queries between test series I and IV, but only a factor of 4 in the resulting optimal scene sizes. Additionally, the graphs for the cost functions are very flat close to their minimum. Thus, we chose 77 KByte as a nearly optimal scene size for all test series.

\section{Conclusion}

In table 5, the access cost for all three models is presented. The cost for model 1 is standardized to " 1 ". For the other two models the numbers describe the speed up factor for 
query processing using these models. The average scene size for model 3 is 79,027 Bytes.

\begin{tabular}{|l|r|r|r|r|}
\hline \multicolumn{1}{|c||}{ model } & \multicolumn{4}{c|}{ speed up factors for query processing } \\
\cline { 2 - 5 } & I $(0.25 \%)$ & II $(1 \%)$ & III $(4 \%)$ & IV $(16 \%)$ \\
\hline \hline 1: Geometry outside of the data pages & 1.0 & 1.0 & 1.0 & 1.0 \\
\hline 2: Geometry inside the data pages & 11.9 & 13.7 & 14.9 & 15.5 \\
\hline 3: Scene organization & 28.9 & 60.5 & 105.7 & 148.5 \\
\hline
\end{tabular}

Tab. 5. Speed up factors for query processing using model 2 and 3 in comparison to model 1

In conclusion, we would like to point out the following statements:

- Storing the exact object representation inside the data pages (model 2) speeds up query processing by a factor of 12 to 15 in comparison to model 1 (using separate pages). The size of the query regions has only a small influence on this factor. For the interpretation of the results one remark is important: The objects used for the tests are relatively small in comparison to the size of the data pages. Using larger objects, i.e. objects larger than one data page, requires storing the exact representation outside of the data pages. As a consequence, query performance of model 2 comes closer to the performance of model 1 .

- The new scene organization is the clear winner of the performance comparison. Even the processing of small queries is performed considerably faster by this storage model. For small queries, we have a speed up factor of about 30 (in comparison to model 1) which is increasing to the impressive value of 148 for large queries.

Another important result is the fact that the optimal scene size is almost independent of the query sizes. Therefore, using the scene architecture with a fixed scene size is beneficial to queries of very different size.

Furthermore, the flat form of the cost function guarantees a considerable speed up of the query processing, even if the average size of the scene is varying caused by insertions and deletions of objects.

\section{Conclusion}

We proposed a storage and access architecture for geographic database systems. This architecture integrates a number of various concepts and techniques for efficient query processing.

The $R^{*}$-tree is the basic component of our geo architecture. It organizes the data on secondary storage with respect to their spatial location and shape. In this way, the search region of spatial queries can be quickly narrowed down. The next ingredient of our architecture are object approximations. They support an efficient preselection to decide whether an object fulfills the query or not. In comparison to the usually used minimum bounding box, the minimum 5-corner is a good compromise between the quality of the approximation and the amount of required storage. The exact geometric representation of an object is managed by a $T R^{*}$-tree. The polygonal objects are decomposed into simpler components and organized with respect to their spatial location and shape. This allows a selective access to the components needed to process a spatial query. Due to the simplicity of the components, the application of time consuming computational geometry algorithms to complex objects is avoided. Thematic queries are supported by 
secondary indices for thematic attributes. These secondary indices are connected to the primary index, i.e. the $\mathrm{R}^{*}$-tree, using a link table.

The parts of our architecture mentioned above support efficient processing of queries with high spatial selectivity, i.e. point queries and small window queries. To speed up the set-oriented object access of large range queries, we added a new ingredient to our architecture: the scene organization. Using this new approach, large parts of the data are combined in scenes and spatially clustered on secondary storage. These scenes are organized within the primary $\mathrm{R}^{*}$-tree. We investigated the performance of this approach in a detailed performance comparison. For large range queries, the scene organization is superior in performance to ordinary storage models with a speed up factor up to two orders of magnitude.

The use of our architecture is not restricted to geographic information systems. With only slight modifications it can also be used in systems for computer aided design (CAD) or computer integrated manufacturing (CIM).

In our future work, we plan to incorporate our geo architecture into an existing extensible database system for spatial applications. Promising candidates for this idea are DASDBS, GRAL and POSTGRES. Performance evaluations of our geo architecture after incorporating it into such a system will be very interesting.

Furthermore the design of a parallel geo architecture is an interesting challenge for future research activities. Parallelism should be exploited in two ways. First, we want to use a multi processor system to process queries in main memory in a massively parallel way. Using object decomposition techniques in a parallel environment promises a considerable performance improvement. Second, we want to use multi disk systems to organize the large data volume of geographic applications more efficiently. The main problem to solve is, to determine an appropriate distribution of the data over the different disk drives.

The application of the presented techniques to $3 D$-objects is another interesting field of research activities for the future. For example, bio-computing is an important field of application for 3D-spatial objects. The first step in this direction is the development and implementation of 3D-approximation and decomposition techniques.

\section{References}

[Aro 91] Aronoff S.: 'Geographic Information Systems', WDL Publications, 1991.

[Bar 88] Bartelme N.: 'GIS Technology: Geographic information systems, land information systems and their fundamentals' (in German), Springer, 1988.

[BKS 93a] Brinkhoff T., Kriegel H.-P., Schneider R.: 'Comparison of Approximations of Complex Objects used for Approximation-based Query Processing in Spatial Database Systems', Proc. 9th Int. Conf. on Data Engineering, Vienna, Austria, 1993.

[BKS 93b] Brinkhoff T., Kriegel H.-P., Schneider R.: 'Scene Organization: A Technique for Global Clustering in Spatial Database Systems', 1993, submitted for publication.

[BKSS 90] Beckmann N., Kriegel H.-P., Schneider R., Seeger B.: 'The R*-tree: An Efficient and Robust Access Method for Points and Rectangles', Proc. ACM SIGMOD Int. Conf. on Management of Data, Atlantic City, NJ., 1990, pp. 322-331.

[Bur 86] Burrough P.A.: 'Principles of Geographical Information Systems for Land Resources Assessment', Oxford University Press, 1986.

[Bur 89] Bureau of the Census: 'TIGER/Line Percensus Files, 1990 Technical Documentation', Washington, DC., 1989. 
[CDRS 86] Carey M. J., DeWitt D. J., Richardson J. E., Shekita E. J.: 'Object and File Management in the EXODUS Extensible Database System', Proc. 12th Int. Conf. on Very Large Data Bases, Kyoto, Japan, 1986, pp. 91-100.

[Cra 90] Crain I.K.: 'Extremely Large Spatial Information Systems - A Quantitative Perspective', Proc. 4th Int. Symp. on Spatial Data Handling, Zürich, Switzerland, 1990, pp. 632-641.

[Fra 91] Frank, A.U.: 'Properties of Geographic Data', Proc. 2nd Symp. on Large Spatial Databases, Zürich, Switzerland, 1991, in: Lecture Notes in Computer Science, Vol. 525, Springer, 1991, pp. 225-234.

[GC 87] Gorny A.J., Carter R.: 'World Data Bank II: General users guide', Technical report, U.S. Central Intelligence Agency, Washington, 1987.

[Gut 84] Guttman A.: 'R-trees: A Dynamic Index Structure for Spatial Searching', Proc. ACM SIGMOD Int. Conf. on Management of Data, Boston, MA., 1984, pp. 47-57.

[Güt 89] Güting R. H.: 'Gral: an extensible relational database system for geografic applications', Proc. 15th Int. Conf. on Very Large Data Bases, Amsterdam, Netherland, 1989, pp. 33-44.

[HS 92] Hoel E.G., Samet H.: 'A Qualitative Comparison Study of Data Structures for Large Line Segment Databases', Proc. SIGMOD Conf., San Diego, CA., 1992, pp 205-214.

[HSW 88] Hutflesz A., Six H.-W., Widmayer P.: 'Globally Order Preserving Multidimensional Linear Hashing', Proc. 4th Int. Conf. on Data Engineering, Los Angeles, CA., 1988, pp. 572-579.

[HWZ 91] Hutflesz A., Widmayer P., Zimmermann C.: 'Global Order Makes Spatial Access Faster', Int. Workshop on Database Management Systems for Geographical Applications, Capri, Italy, 1991, in: Geographic Database Management Systems, Springer, 1992, pp. 161-176.

[KBS 91] Kriegel H.-P., Brinkhoff T., Schneider R.: 'An Efficient Map Overlay Algorithm based on Spatial Access Methods and Computational Geometry', Int. Workshop on Database Management Systems for Geographical Applications, Capri, Italy, 1991, in: Geographic Database Management Systems, Springer, 1992, pp. 194-211.

[KHS 91] Kriegel H.-P., Horn H., Schiwietz M.: 'The Performance of Object Decomposition Techniques for Spatial Query Processing', Proc. 2nd Symp. on Large Spatial Databases, Zürich, Switzerland, 1991, in: Lecture Notes in Computer Science, Vol. 525, Springer, 1991, pp. 257-276.

[Kri 91a] Kriegel H.-P., Heep P., Heep S., Schiwietz M., Schneider R.: 'An Access Method Based Query Processor for Spatial Database Systems', Int. Workshop on Database Management Systems for Geographical Applications, Capri, Italy, 1991, in: Geographic Database Management Systems, Springer, 1992, pp. 273-292.

[Kri 91b] Kriegel H.-P., Heep P., Heep S., Schiwietz M., Schneider R.: 'A Flexible and Extensible Index Manager for Spatial Database Systems', Proc. 2nd Int. Conf. on Database and Expert Systems Applications, Berlin, Germany, 1991, pp. 179-184.

[Ore 89] Orenstein J. A.: 'Redundancy in Spatial Databases', Proc. ACM SIGMOD Int. Conf. on Management of Data, Portland, USA, 1989, pp. 294-305.

[PH 90] Paterson D., Hennessy J.: 'Computer Architecture: A Quantitative Approach', Morgan Kaufman, 1990.

[PS 88] Preparata F.P., Shamos M.I.: 'Computational Geometry', Springer, 1988.

[Sam 90] Samet H.: 'The Design and Analysis of Spatial Data Structures', Addison Wesley, 1990.

[Sch 92] Schneider R.: 'A Storage and Access Structure for Spatial Database Systems', Ph.D.-thesis (in German), Institute for Computer Science, University of Munich, 1992. 
[SK 91] Schneider R., Kriegel H.-P.: 'The TR*-tree: A New Representation of Polygonal Objects Supporting Spatial Queries and Operations', Proc. 7th Workshop on Computational Geometry, Bern, Switzerland, 1991, in: Lecture Notes in Computer Science, Vol. 553, Springer, 1991, pp. 249-264.

[SR 86] Stonebraker M., Rowe L.: 'The Design of POSTGRES', Proc. ACM SIGMOD Conf. on Management of Data, Washinton D.C., 1986.

[SV 89] Scholl M., Voisard A.: 'Thematic Map Modelling', Proc. 1st Symp. on the Design and Implementation of Large Spatial Databases, Santa Barbara, CA., 1989, in: Lecture Notes in Computer Science, Vol. 409, Springer, 1990, pp. 167-190.

[SW 86] Schek H.-J., Waterfeld W.: 'A Database Kernel System for Geoscientific Applications', Proc. 2nd Int. Symp. on Spatial Data Handling, Seattle, Washington, 1986, pp. 273-288.

[Wei 89] Weikum G.: 'Set-Oriented Disk Access to Large Complex Objects', Proc. 5th Int. Conf. on Data Engineering, Los Angeles, CA., 1989, pp. 426-433.

[Wid 91] Widmayer P.: 'Data Structures for Spatial Databases' (in German) in: Vossen G., Witt K.-U. (eds.): 'Entwicklungstendenzen bei Datenbank-Systemen' (Future Trends in Database Systems), Oldenbourg, 1991, pp. 317-361. 